\title{
Diagnostic Utility of BRCA1-Associated Protein 1 (BAP1) in Distinguishing Reactive and Neoplastic Mesothelial Proliferation
}

\author{
Rasha M. El-Sawy, Hadeer M. Elshaha, Hala A. Agina, Sahar E. Mohamed, Heba M. Rashad
}

\begin{abstract}
Department of Pathology, Benha faculty of medicine, Benha University, Egypt.

\section{Correspondence to: Hadeer M. Elshaha. Department of pathology, Benha faculty of medicine, Benha University, Egypt.}

\section{Email:}

dodda111190@gmail.com

Received: 12 October 2020

Accepted: 1 November 2020

\section{Abstract:}

Background: Malignant mesothelioma (MM) is an aggressive tumor which arises from the lining of serous cavities. Distinguishing between begnin and malignant mesothelial proliferation on effusions is a challenge. This study aims to evaluate the diagnostic utility of BAP1 in distinguishing MM from reactive mesothelial proliferation. Material and methods: This is a retrospective study on 61 cases; pleural biopsies $(n=36)$ and pleural cell blocks $(n=25)$. Included were synchronous cytology/ biopsy pair samples. All cases were stained with BAP-1antibody using avidin-biotin complex. BAP1 immunohistochemistry was evaluated using cut off value; negative staining indicate malignancy. Statistical analysis was done using SPSS (version 20), $\mathrm{P}$ value ( $\mathrm{P}$ value $<0.05$ was considered statistically significant). ROC curve to predict cut off value. Results: In MM cases $61.1 \%$ showed BAP1 negative nuclear expression. In reactive mesothelial proliferation cases; $20 \%$ showed BAP1 negative expression. In atypical mesothelial proliferation cases, $80 \%$ showed BAP1 negative nuclear expression. synchronous cytology/ biopsy pairs (13 cases) showed BAP1 matching results. There was a highly statistically significant correlation between BAP1 expression and the study groups (P-value <0.001). There was statistically significant correlation between BAP-1 expression and histological types of mesothelioma (P-value <0.05) and stage (P-value <0.05). In cell blocks; sensitivity was $80 \%$ and specificity was $80 \%$ for atypical mesothelial proliferation. In tissue biopsy; sensitivity was $61.1 \%$ and Specificity was $80 \%$ for mesothelioma. Conclusion: In effusions, negative BAP1 strongly support the diagnosis of malignant mesothelioma, so BAP1 may be included in immunohistochemical panels for malignant mesothelioma cytodiagnosis.

Keywords: malignant mesothelioma, reactive mesothelial proliferation, BAP1 immunohistochemistry. 



\section{Introduction:}

Malignant mesothelioma (MM) is an aggressive tumor that arises from mesothelial cells which form the lining of the pleural, pericardial, and peritoneal cavities (1).

Majority of malignant mesothelioma occur in pleural cavity while most of the remaining occur in peritoneal cavity (2). According to the National Cancer Institute's Surveillance, Epidemiology and End Results (SEER) database, annual incidence rates for mesothelioma are holding steady around one new case per 100,000 people (3).

In the United States (U.S) around 3,000 new cases are diagnosed each year (4). In Egypt pleural malignant tumors formed $1.28 \%$ of total malignant tumors. Pleural mesothelioma is the most common primary malignant tumor forming more than half of the cases (5).

The main risk factor for pleural mesothelioma is exposure to asbestos. Some individuals develop malignant mesothelioma following exposure to small amounts of asbestos, whereas others exposed to heavy amounts do not, suggesting that genetic factors influence risk of this disease. Other risk factors may include; radiation exposure, being older, being male and exposure to certain other mineral (6). Malignant mesothelioma is identified by three cell types that compose tumors; epithelioid, sarcomatoid and biphasic (7). Patient present clinically by cough, chest pain, difficulty breathing, pleural nodules, diffuse pleural thickening and pleural effusion (8).

Staging describes the anatomical extent of a tumour and the classification based on TNM staging system established by the International Mesothelioma Interest Group (IMIG) and the International Association for the Study of Lung Cancer (IASLC) (9).

The nuclear grading system for epithelioid mesothelioma based on the two independent prognostic factors: nuclear atypia and mitotic count (10).

Prognosis is very poor with median survival of 9-12 months of pleural cases and could be affected by histological subtype, age and gender. Epithelioid subtype associated with the best prognosis and the sarcomatoid subtype with the worst (11). Distinguishing between begnin and malignant mesothelial proliferation on effusions is a challenge (12).

BAP-1 is tumour suppressor gene located on chromosome $3 \mathrm{p} 21$ and functions as a deubiquitinating enzyme, specifically regulating chromatin remodeling, functioning 
as a mediator of DNA damage responses and growth suppression (13).

BAP-1 plays a role in modulation of calciuminduced apoptosis, so mutation may result in accumulation of DNA-damaged cells and greater susceptibility to development of malignancy (14).

Germline mutations in BAP-1 have been observed in families with a high frequency of malignant mesothelioma and was associated with earlier age of onset (15). Mutations in BAP1 gene also occur in other tumors like uveal melanoma, begnin atypical melanocytic lesions, cutaneous melanoma, renal cell carcinoma, cholangiocarcinoma and basal cell carcinoma (16).

\section{Aim of the work:}

This study aims to evaluate the diagnostic utility BAP-1 immunohistochemistry in distinguishing between reactive and atypical mesothelial cells in pleural effusion and correlate the results to clinico-pathological findings.

\section{Material and methods:}

This is retrospective study carried on 61 cases; 36 cases of pleural biopsies and 25 cases of pleural cell blocks; thirteen cases have both pleural biopsies and pleural effusions. Out of the 25 pleural effusions cell blocks, 10 cases were atypical mesothelial proliferation and 15 cases were reactive mesothelial proliferation. This study was performed on archival formalin fixed paraffin embedded blocks which were collected from Benha Pathology Department and Early Cancer Detection Unit (ECDU), Faculty of Medicine, Benha University proved by ethical committee. They were collected from January 2013 to December 2017. All the original histologic slides were reviewed by two pathologists; they were blinded to patient identity and initial tumor categorization to ensure tumor consistency. Cases were graded into low grade (G I) and high grade (G II and III) tumors according to Kadota et al., (2012)(10) and staged according to Berzenji et al., (2018)(17). Sarcomatoid and biphasic types were gathered into non epithelioid group for statistical analysis since they are both considered high grade. Stage I and II were gathered as low stage and stage III and IV were gathered as high stage for statistical analysis.

For Immunohistochemical studies: avidinbiotin complex technique was used following manufacture instructions (Dako, CA). For antigen retrieval citrate monohydrate $(\mathrm{pH}$ 6.0) was used. Slides were incubated with an anti-BAP1 antibody (Abbexa Ltd Innovation 
Centre, Cambridge Science Park, Cambridge, CB4 OEY, UK) at 1:200 dilution, for 1 hour at room temperature. Freshly prepared chromogen diaminobenzine (DAB) was used; it was incubated with slides for 3-5 minutes. In each staining session sections of breast carcinoma was used as positive control for BAP-1. For negative control, the primary antibody was omitted and replaced by normal rabbit serum IgG. At the time of interpreting the immunohistochemistry, the observers were blinded to the underlying diagnosis. All immunostained slides were examined by 2 observers, the candidate and supervisor for confirmation.

\section{Immunohistochemical interpretation:}

Sections were evaluated under light microscope and only nuclear BAP-1 expression is regarded. The extent of immunostaining was evaluated in random 4 fields under the power of 200 magnification. According to cut off value in our results, the results were expressed in histologic section as; positive staining when $>57.5 \%$ of target cells show nuclear immunoreactivity, negative staining when $<57.5 \%$ showed immune-reactivity. The results were expressed in cell blocks as; positive staining by pleural effusion were confirmed by biopsy to be malignant mesothelioma. Out of 15 cases of reactive mesothelial proliferation, 3 cases were found to be malignant mesothelioma, by when $>32.5 \%$ of target cells show immunereactivity, negative staining when $<32.5 \%$ showed nuclear immune-reactivity. Negative nuclear BAP1 expression indicate malignancy. Cases with cytoplasmic staining were disregarded (18).

Statistical analysis: Results were analyzed using the computer program Statistical package for social science (SPSS version 20 for windows; SPSS Inc., Chicago, Illinois, USA). ROC curve used to predict the cutoff point of BAP-1.

Statistically significance of the tests were expressed in P-value. A P value $<0.05$ was considered statistically significant. $\mathrm{P}$ value $<0.01$ was considered highly significant.

\section{Results}

Out of studied 61 cases; 36 cases are mesothelioma pleural biopsies and 25 cases are pleural cell blocks, 13 cases have both pleural biopsies and pleural effusions. Out of the 25 pleural effusions cell blocks, 10 cases were atypical mesothelial proliferation and 15 cases were reactive mesothelial proliferation. The atypical cases diagnosed

biopsy. In all cases; forty seven cases were males and 14 cases were female. The mean age was 55.9 \pm 12.51 . Clinicopathological data are shown in Table (1). 


\section{Histopathological results :}

There was a statistically significant correlation between histologyical types with presentation by pleural effusion (P-value <0.05) and clinical presentation with diffuse pleural thickning or pleural nodules (P-value $<0.05$ ). No statistically significant correlation between histologyical types with staging (P-value $>0.05$ ) and grading ( $\mathrm{P}$-value $>0.05)$.

Immunohistochemical results : shown in Table (1)

There was statistically significant correlation between BAP1 expression and reactive mesothelial proliferation, atypical mesothelial proliferation and mesothelioma ( $\mathrm{P}$ value $<0.001)$. Figure (1)

There was statistically significant correlation between BAP-1 expression with histological types of mesothelioma (P-value <0.05) and the stage (P-value <0.05). No statistically significant correlation between BAP-1 expression and the grade $(\mathrm{P}$-value $>0.05)$ was detected.

By using ROC curve: in tissue biopsy, AUC was 0.92 , sensitivity was $61.1 \%$ and specificity was $80 \%$, cut off value of BAP-1 expression was $57.5 \%$, Positive predictive value was $88 \%$ and negative predictive value was $46.1 \%$ for mesothelioma.

By using ROC: in cell blocks, AUC of BAP-1 was 0.95 , Sensitivity was $80 \%$ and Specifity was $80 \%$, Cut off value of BAP-1 expression was $32.5 \%$, positive predictive value was $72.5 \%$ and negative predictive value was $85.5 \%$ for atypical mesothelial proliferation. Graph (1)

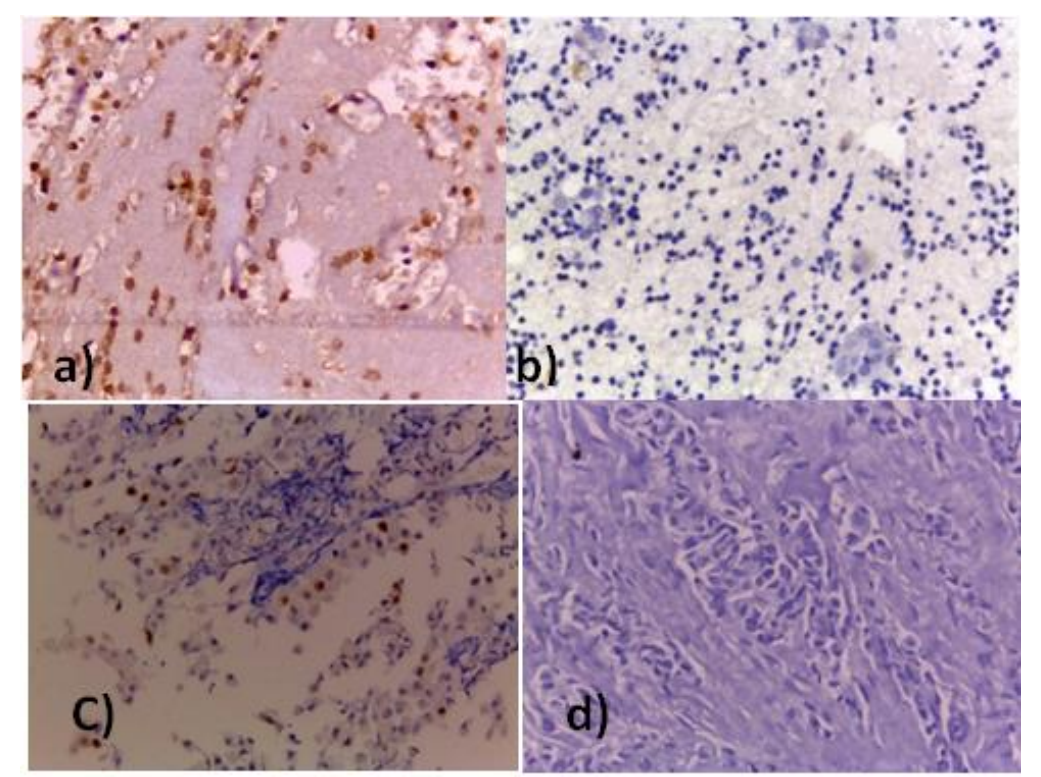

Figure (1): (a) BAP1 staining cell block preparation: reactive mesothelial proliferation; positive nuclear BAP1 expression in scattered mesothelial cells and positive lymphocytes (internal control) (b) BAP1 staining cell block preparation: atypical mesothelial proliferation, negative nuclear BAP1 expression in mesotheliomatous clusters. CBAP1 staining in malignant mesothelioma; tissue section: positive nuclear BAP1 expression in some mesotheliomatous cells. (d) BAP1 staining in malignant mesothelioma; tissue section: negative nuclear BAP1 expression in mesotheliomatous cells. (ABC X 400) 
Benha medical journal vol. 38, academic issue, 2021

Table 1: Clinicopathological data of studied groups.

\begin{tabular}{|c|c|c|c|c|}
\hline & $\begin{array}{l}\text { Pleural biopsy } \\
\text { Mesothelioma group (36) }\end{array}$ & $\begin{array}{l}\text { Pleural effusion } \\
\text { Reactive group (15) }\end{array}$ & Atypical group (10) & $P$ value \\
\hline \multicolumn{5}{|l|}{ Sex } \\
\hline Male & $27(75 \%)$ & $12(80 \%)$ & $8(80 \%)$ & \\
\hline Female & $9(25 \%)$ & $3(20 \%)$ & $2(20 \%)$ & \\
\hline Age /year & $58.39 \pm 12.49$ & $49.73 \pm 12.69$ & $56.2 \pm 9.93$ & \\
\hline Pleural thicknning & & - & - & \\
\hline Nodules & $18(50 \%)$ & & & \\
\hline Diffuse & $18(50 \%)$ & & & \\
\hline Pleural effusion & & - & - & \\
\hline Present & $25(69.4 \%)$ & & & \\
\hline Absent & $11(30.6 \%)$ & & & \\
\hline \multicolumn{5}{|l|}{ H\&E } \\
\hline Epitheloid & $22(61.1 \%)$ & & & \\
\hline Non epithelioid & $14(38.9 \%)$ & & & \\
\hline \multicolumn{5}{|l|}{ Grade } \\
\hline Low & $5(13.9 \%)$ & & & \\
\hline High & $31(86.1 \%)$ & & & \\
\hline Stage & & - & - & \\
\hline Low & $15(41.7 \%)$ & & & \\
\hline High & $21(58.3 \%)$ & & & \\
\hline BAP1 & & & & $<0.001 *$ \\
\hline Positive & $14(38.9 \%)$ & $12(80 \%)$ & $2(20 \%)$ & \\
\hline negative & $22(61.1 \%)$ & $3(20 \%)$ & $8(80 \%)$ & \\
\hline
\end{tabular}

*highly significant

\section{Discussion:}

Malignant pleural mesothelioma is an aggressive cancer that develops in the pleura and it is the most common site among the four sites of mesothelioma (19). According to National Cancer Institute about 3,000 new cases of mesothelioma are diagnosed in the US each year, more often in men, those aged
65 years and older (3). In Egypt, according to the National Cancer Institute, pleural mesothelioma was the most common primary pleural malignant tumor forming more than half the cases (53.08\%). Epithelioid type mesothelioma was the most frequent histologic subtype forming76.20\% of all mesotheliomas (5). 
It is difficult to distinguish reactive mesothelial proliferation from malignant mesothelioma especially in effusion. A new marker may aid in distinguishing malignant mesothelioma (MM) from reactive mesothelial proliferation, namely BRCA1associated protein 1 (BAP1) (12).

The current work showed that the mean age of mesothelioma cases was 58.4 (range from 36-84 years) which is close to the study performed by others (20) who stated that the mean age of patients was 52.1 years (range from 24-78 years).

On the other hand, the age distribution is different from the data derived from many Western studies, where most patients are diagnosed at age 65 or older. According to the latest SEER report (2014)(21), people aged 80-84 have the highest mesothelioma incidence. This may be explained by different number of cases, variable exposure to environmental conditions and incidence increased by age.

In our work, $75 \%$ of mesothelioma cases are males and $25 \%$ are females.

This was in line with the study performed by other researchers (22) who indicated that there is increase in the incidence of pleural mesothelioma especially for men because of the exposure to asbestos. Same results were obtained before (23)

On the other hand, the study performed by the group of researchers on 2011 (20) showed that the male: female (M: F) ratio was 1:1. The difference may be due to all female cases came from endemic areas.

The current work showed that $69.4 \%$ of mesothelioma cases presented with pleural effusion. These results were consistent with the study performed previously (24) which showed that up to $95 \%$ of patients suffer from a pleural effusion during their disease course. Similar finding by were obtained by a group of researchers $(25,26)$ who stated that malignant pleural effusion (MPE) occurs in $54-90 \%$ of all malignant pleural mesothelioma cases which indicated that $83 \%$ of mesothelioma had a pleural effusion at presentation. These results showed that malignant pleural effusion (MPE) is the commonest presentation of mesothelioma.

BAP-1 is tumour suppressor gene located on chromosome 3 p21 and functions as a deubiquitinating enzyme, which mutation may play role in malignant transformation (13). Concerning immunohistochemical results, statistical analysis receiver operating characteristic (ROC) curve analysis was performed to establish the cut-off value. The results were expressed in mesothelioma as; 
positive staining when $>57.5 \%$ of target cells show immunoreactivity, negative staining when $<57.5 \%$ showed immune-reactivity. The results were expressed in cytology as; positive staining when $>32.5 \%$ of target cells show immune-reactivity, negative staining when $<32.5 \%$ showed immune-reactivity.

There were some studies which dealt with the cutoff issue. One define negative staining when $>50 \%$ of target cells show loss of immunoreactivity. (27), the other proposed a score according to the percentage of positive tumor cells: 0 (<10\%), $1(10 \%-49 \%), 2$ (50\%-90\%), 3 (>90\%). (28). In other studies, the expression of BAP1 is described as negative or positive as the studies.(29) and (18).

The difference in the cutoff value may be explained by different sample size, different marker antibody or technique used. In our work, out of 15 cases of reactive mesothelial proliferation $80 \%$ were positive BAP1 nuclear expression and $20 \%$ were BAP1 negative nuclear expression, which were proved in biopsy examination to be mesothelioma. This discordance in the results of cytology and biopsy explained that the atypia in negative BAP1 cases is low so the diagnosis by $\mathrm{H} \& \mathrm{E}$ may not be accurate $100 \%$ (27). Another explanation, that in a fraction of mesotheliomas, loss of BAP1 protein might represent an early and irreversible event anticipating full mesothelial transformation (12).

Out of 10 cases of atypical mesothelial proliferation $80 \%$ were negative BAP1 nuclear expression and $20 \%$ were positive BAP1 nuclear expression, which confirmed by biopsy examination to be mesothelioma. The positive cases may be explained that not all cases of mesothelioma occur due to BAP1 mutation. Another explanation for this positive cases that they may be due to sarcomatoid type as sarcomatoid type generally exhibit less shedding of cells into pleural space (30).

The current work illustrated that there is highly significant correlation between BAP-1 expression and reactive mesothelial hyperplasia, atypical mesothelial proliferation and mesothelioma (P-value <0.001). BAP-1 negative expression in $61.1 \%$ of mesothelioma and $80 \%$ of atypical mesothelial proliferation and retained in $80 \%$ of reactive mesothelial proliferation, which indicate that negative expression of BAP1 may contributes to malignant transformation. This was explained by the fact that BAP-1is tumour suppressor gene. (31). 
The BAP1 expression by

immunohistochemistry represents a

biomarker of excellent clinical utility for the diagnosis of malignant mesothelioma being lost in $66 \%$ of mesotheliomas.(12) Our results were consistent with the study performed before in which it was stated that negative BAP1 expression was found in $47.5 \%$ in mesothelioma.(18)

It was stated that BAP1 loss seems to be exclusively seen in malignant lesions. As loss of BAP1 expression was found in $59 \%$ on malignant mesothelioma cases. (32) Similar finding were proved by other researchers (33, $34)$.

The difference in BAP-1 negativity percentage may be explained by different number of cases and different cut off value used.

The current work showed $77.3 \%$ of epithelioid mesothelioma was negative nuclear BAP1 expression and $35.7 \%$ of nonepithelioid mesothelioma were negative nuclear BAP1 expression. There was statistically significant correlation between BAP-1 expression and histological types of mesothelioma (P-value <0.05).

The percentage of negative cases was higher in epithelioid mesothelioma. This may be explained by higher number of cases or BAP1 mutation is more common in epithelial type than non-epithelial type (35).
It was proved that negative BAP1 expression was evident mainly in epithelioid type of mesothelioma.(36)

It was established that BAP1 protein was lost in a large proportion of mesotheliomas, especially with epithelioid type (70\%) and biphasic types (60\%). BAP1 loss was also seen in sarcomatoid and desmoplastic mesothelioma (15\%), although with lower frequency.(12, 29)

This was also consistent with the study that confirmed that the difference in BAP1 negativity between epithelial and nonepithelial variants (71\% vs $10 \%)$. (18)

From all previous results BAP1 could be useful in the diagnosis of epithelioid type MM, because the lack of the tumor suppressor BAP1 may be more specifically involved in the pathogenesis of epithelioidtype MM rather than non-epithelioid MM (36).

The current work revealed that BAP-1 expression has no statistically significant correlation with grading of mesothelioma (Pvalue $>0.05)$.

Up to our knowledge, there was no previous study illustrated the relationship between BAP-1 and the grade of mesothelioma. On the contrary, a study established that BAP1 mutated colorectal and renal carcinomas are 
associated with high tumor grading $(\mathrm{P}<0.0001) .(37)$ Also another study indicated that BAP1 deficient in clear cell renal cell carcinoma were of high grade.(18)

This discrepancy could be explained by different functions of BAP-1 depending on the tissue in which they are expressed. This work showed that BAP-1 expression has statistically significant correlation with stage of mesothelioma (P-value <0.05).

Up to our knowledge, there were no previous studies illustrated the relationship between BAP-1 and the stage of mesothelioma. It was concluded before that nuclear BAP1-negative RCC had higher tumor size, higher Fuhrman grade, and higher stage, a greater amount of vascular and capsular invasion and a higher incidence of metastases (39). Also another study on intrahepatic cholangiocarcinoma established that patients with BAP1 loss were less likely to present with more advanced tumors or with lymphatic invasion, but were more likely to present with larger tumors. (33)

The current work showed the sensitivity of BAP-1 was $80.0 \%$ in atypical mesothelial proliferation and $61 \%$ in mesothelioma.

The sensitivity of BAP1 is higher in effusions may be explained by the fact that most of the atypical shed cells are from the epithelioid type MM, effusions commonly of epithelioid type, as sarcomatoid mesothelioma often does not shed malignant cells into the pleural effusion and may instead induce an overlying reactive mesothelial proliferation (30).

Our results were close to the studies performed on year 2015 (12), where the sensitivity was $66 \%$ and that on year 2016, (40) who cited $67.5 \%$ using BAP1 IHC for MM diagnosis, but different from the studies performed on 2015 (34) who reported a sensitivity $27 \%$ for mesothelioma and that done on 2018 (41) which stated that the sensitivity of BAP1 IHC was $37.1 \%$ for MM diagnosis.

This difference could be because of the higher number of epithelioid mesothelioma cases included in the present study.

The specificity of BAP-1 was $80 \%$ in both pleural effusion and biopsy cases. Our study was close to the study performed before which reported $95 \%$ specificity and that on 2016reported $85.7 \%$ specificity. (33.27)

Other studies reported higher specificity of BAP1 in both pleural effusion and biopsy. For instance, a study done on 2015 (12) was in line with our study, showing the high specificity $(100 \%)$ of BAP1 loss for mesothelioma diagnosis. Similar results were also obtained. $(34,18,41)$. 
This variation could be explainable by a different ways to evaluated immunostaining BAP1 results and the difference in the number of studied sample.

The current work revealed that positive predictive value was 88 and negative predictive value was 46.1 in mesothelioma cases and 72.5 positive predictive value and 85.5 negative predictive value in atypical mesothelial proliferation.

This was close to the study performed on 2015 (12) which also establishes that BAP1 had $100 \%$ positive predictive value for mesothelioma development, whereas $90 \%$ negative predictive value. Similar results were obtained 18) where it was established that, the PPV value (BAP1 staining negative) is $100 \%$.

\section{Conclusion}

BAP1 immunohistochemistry negativity on cytology preparations may be used as a useful tool for distinguishing between malignant versus reactive pleural effusions together with routine preparation. It could be included in IHC panels for MM cytodiagnosis. It may be used to support the diagnosis of malignancy in atypical mesothelial proliferations. The finding of a positive BAP1 IHC result does not exclude a MM diagnosis, because not all MMs may harbor alterations of the BAP1 gene. Also minority of epithelioid MM retained BAP1 staining.

\section{Reference}

1. Carbone M, Ly BH, Dodson RF, Pagano I, Morris PT, Dogan UA et al., (January 2012). Malignant mesothelioma: facts, myths, and hypotheses. Journal of cellular physiology. 227 (1): 44-58.

2. Pass HI, Carbone M, Krung LM, Rosenzweig KE (2015). Benign and malignant mesothelioma. DeVita, Hellman, and Rosenberg's Cancer: Principles and Practice of Oncology. 10th ed. 2015 Philadelphia, Pa: Lippincott Williams \& Wilkins.

3. Panou V, Vyberg M, Weinreich UM, Meristoudis C, Falkmer UG, Røe OD, (June 2015). The established and future biomarkers of malignant pleural mesothelioma. Cancer treatment reviews. 41(6): 486-95.

4. Robinson BM (2012). Malignant pleural mesothelioma: an epidemiological perspective. Ann Cardiothorac Surg.1(4):491.

5. Mokhtar N, Salama A, Badawy O, Khorshed E, Mohamed G, Ibrahim M, et al. (2016). Cancer Pathology Registry 2000-2011. Cairo, Egypt: cairo university. 2016.

6. Comar M, Zanotta N, Pesel G, Visconti P, Maestri I, Rinaldi R et al.,(2012). Asbestos and SV40 in malignant pleural mesothelioma from a hyperendemic area of north-eastern Italy. Tumori Journal. 98(2):210-214.

7. Pasello G, Urso L, Mencoboni M, Grosso F, Ceresoli GL, Lunardi F et al., (2015). MDM2 and HIF1alpha expression levels in different histologic subtypes of malignant pleural mesothelioma, 
correlation with pathological and clinical data, Oncotarget. 6(39):42053.

8. Mercadante S, Degiovanni D, and Casuccio A (2016). Symptom burden in mesothelioma patients admitted to home palliative care. Current Medical Research and Opinion. 32(12):1985-1988.

9. Rusch VW, Chansky K, Kindler HL, Nowak A K, Pass H I, Rice D C et al. (2016). The IASLC Mesothelioma Staging Project: proposals for the M descriptors and for revision of the TNM stage groupings in the forthcoming (eighth) edition of the TNM classification for mesothelioma. J Thorac Oncol.;11:2112-2119.

10. Kadota K, Suzuki K, Colovos C, Sima C S, Rusch V W, Travis W D et al., (2012). A nuclear grading system is a strong predictor of survival in epitheloid diffuse malignant pleural mesothelioma. Modern Pathology, 25(2), 260.

11. Leuzzi G, Rea F, Spaggiari L, Faccolo F (2015). Prognostic score of long-term survival after surgery for malignant pleural mesothelioma: A multicenter analysis. Ann Thorac Surg. 100(3):890-897.

12. Cigognetti M, Lonardi S, Fisogni S, Balzarini P, Pellegrini V, Tironi A, et al (2015). BAP1 (BRCA1-associated protein 1) is a highly specific marker for differentiating mesothelioma from reactive mesothelial proliferations. Modern Pathology, 28(8): 1043.

13. McGregor S M, Dunning R, Hyjek E, Vigneswaran W, Husain A N, and Krausz T(2015). BAP1 facilitates diagnostic objectivity, classification, and prognostication in malignant pleural mesothelioma. Human Pathology 46(11):1670-1678.

14. Bononi A, Giorgi C, Patergnani S, Larson D, Verbruggen K, Tanji M, et al (2017). BAP1 regulates IP3R3-mediated $\mathrm{Ca}^{2+}$ flux to mitochondria suppressing cell transformation. Nature, 546(9659):549-553.

15. Baumann F, Flores E, Napolitano A, Kanodia $S$ (2015). Mesothelioma patients with germline BAP1 mutations have 7-fold improved long-term survival. Carcinogenesis, 36(1,):76-81.

16. Murali R, Wiesner T, and Scolyer RA (2013). Tumours associated with BAP1 mutations. Pathology. 45(2):116-126.

17. Berzenji, L., Van Schil, P. E., \& Carp, L. (2018). The eighth TNM classification for malignant pleural mesothelioma. Translational lung cancer research, 7(5), 543.

18. Cozzi I, Oprescu FA, Rullo E, \& Ascoli V, (2018). Loss of BRCA1-associated protein 1 (BAP 1) expression is useful in diagnostic cytopathology of malignant mesothelioma in effusions. Diagnostic cytopathology 46(1):9-14.

19. Kondola S, Manners D, Nowak AK (February 2016). Malignant pleural mesothelioma: an update on diagnosis and treatment options. Therapeutic Advances in Respiratory Disease. 10(3):275-88.

20. Adel AM, Abdel Hafeez ZM, El Sheikh ET, El Sharawy IA, \& Gobran NS (2011). Malignant pleural mesothelioma: a retrospective analysis of clinicopathological and survival data. Thoracic cancer. 2(1):16-23.

21. Beebe-Dimmer JL, Fryzek JP, Yee CL, Dalvi TB, Garabrant DH, Schwartz AG, et al (2016). Mesothelioma in the United States: a surveillance, epidemiology, and end results (SEER)-Medicare investigation of treatment patterns and overall survival. Clinical epidemiology. 8:743.

22. Jarvholm B, and Burdorf A (2015). Emerging evidence that the ban on asbestos use is reducing the occurrence of pleural mesothelioma in Sweden. Scand J Public Health. 43: 875-881. 
23. Soeberg MJ, Leigh J, Driscoll T, Armstrong B, Young JM, \& van Zandwijk N. (2016). Incidence and survival trends for malignant pleural and peritoneal mesothelioma, Australia, 19822009. Occup Environ Med, 73(3), 187-194.

24. Cheah HM, Lansley SM, Varano della Vergiliana JF, Tan AL, Thomas R, Leong SL, et al., (2017). Malignant pleural fluid from mesothelioma has potent biological activities. Respirology, 22(1), 192-199.

25. Arnold DT and Maskell NA (2018). Biomarkers in mesothelioma. Ann. Clin. Biochem. $55,49-58$.

26. Skok K, Hladnik G, Grm A, \& Crnjac A. (2019). Malignant Pleural Effusion and Its Current Management: A Review. Medicina, 55(8), 490

27. Walts AE, Hiroshima K, McGregor SM, Wu D, Husain AN, \& Marchevsky AM. (2016). BAP1 immunostain and CDKN2A (p16) FISH analysis: clinical applicability for the diagnosis of malignant mesothelioma in effusions. Diagn Cytopathol, 44:599-606.

28. Shinozaki-Ushiku A, Ushiku T, Morita S, Anraku M, Nakajima J, \& Fukayama M. (2017). Diagnostic utility of BAP1 and EZH2 expression in malignant mesothelioma. Histopathology 2017;70:722-733.

29. Righi L, Duregon E, Vatrano S, Izzo S, Giorcelli J, Rondón-Lagos M, et al (2016). BRCA1-associated protein 1 (BAP1) immunohistochemical expression as a diagnostic tool in malignant pleural mesothelioma classification: a large retrospective study. Journal of Thoracic Oncology, 11(11):2006-2017.

30. Muruganandan S, Alfonso H, Franklin P, Shilkin K, Segal A, Olsen N,.,et al., (2017). "Comparison of outcomes following a cytological or histological diagnosis of malignant mesothelioma," British Journal of Cancer, vol. 116 (6), 703-708.

31. Wang A, Papneja A, Hyrcza M, Al-Habeeb A and Ghazarian D (2016). Gene of the month: BAP1. J Clin Pathol 2016;69(9):750-753.

32. Pulford E, Huilgol K, Moffat D, Henderson DW, \& Klebe S (2017). Malignant mesothelioma, BAP1 immunohistochemistry, and VEGFA: does BAP1 have potential for early diagnosis and assessment of prognosis? Disease markers 2017.

33. Andrici J, Sheen A, Sioson L, Wardell K, Clarkson A, Watson N, et al (July 2015). Loss of expression of BAP1 is a useful adjunct, which strongly supports the diagnosis of mesothelioma in effusion cytology. Mod Pathol. 28(10):1360-1368.

34. Sheffield BS, Hwang HC, Lee AF, Thompson $\mathrm{K}$, Rodriguez $\mathrm{S}$, Tse $\mathrm{CH}$, et al (2015). BAP1 immunohistochemistry and p16 FISH to separate benign from malignant mesothelial proliferations. Am J Surg Pathol. 39:977-982.

35. Nasu M, Emi M, Pastorino S, Tanji M, Powers A, Luk H,et al. (2015). High incidence of somatic BAP1 alterations in sporadic malignant mesothelioma. J Thorac Oncol;0:565-576.

36. Yoshikawa Y, Sato A, Tsujimura T, Emi M, Morinaga T, Fukuoka K, et al, (2012). Frequent inactivation of the BAP1 gene in epithelioid type malignant mesothelioma. Cancer Sci. 103:868874.

37. Luchini C, Veronese N, Yachida S, Cheng L, Nottegar A, Stubbs B, et al, (2016). Different prognostic roles of tumor suppressor gene BAP1 in cancer: A systematic review with metaanalysis. Genes, Chromosomes and Cancer, 55(10): 741-749.

38. Gu YF, Cohn S, Christie A, McKenzie T, Wolff N, Do QN, et al. (2017). Modeling renal cell 
carcinoma in mice: Bap1 and Pbrm1 inactivation drive tumor grade. Cancer discovery, 7(8):900-917.

39. Minardi D, Lucarini G, Milanese G, Di Primio R, Montironi R, \& Muzzonigro G, (Augst 2016). Loss of nuclear BAP1 protein expression is a marker of poor prognosis in patients with clear cell renal cell carcinoma. In Urologic Oncology: Seminars and Original Investigations, 34(8):338e11).

40. Hida T, Hamasaki M, Matsumoto S, Sato A, Tsujimura T, Kawahara K, et al, (2016). BAP1 immunohistochemistry and p16 FISH results in combination provide higher confidence in malignant pleural mesothelioma diagnosis: ROC analysis of the two tests. Pathol Int, 1:1-8.

41. Liu J, Liao X, Gu Y, Fu L, Zhao J, Li L, et al., (2018). Role of p16 deletion and BAP1 loss in the diagnosis of malignant mesothelioma. Journal of thoracic disease, 10(9), 5522.

To cite this article: Rasha M. El-Sawy , Hadeer M. Elshaha, Hala A. Agina, Sahar E. Mohamed, Heba M. Rashad. Diagnostic Utility of BRCA1-Associated Protein 1 (BAP1) in Distinguishing Reactive and Neoplastic Mesothelial Proliferation. BMFJ 2021; 38 (academic issue): 47-60, DOI: 10.21608/bmfj.2020.45981.1327 